\title{
Quasi-Equivalence of Quasi-Free States on the Weyl Algebra
}

\author{
A. VAN DAELE ${ }^{\star}$ \\ Institute of Mathematics, University of Oslo, Oslo
}

Received November 5, 1970

\begin{abstract}
A necessary and sufficient condition for quasi-equivalence of quasi-free factor states over the Weyl algebra is proved. The essential part of this paper is closely related to the work of Powers and Størmer on the Clifford algebra.
\end{abstract}

\section{Introduction}

In this paper we study the quasi-equivalence of quasi-free states of the canonical commutation relations. It is well known that all irreducible representations of these relations for finite systems are unitarily equivalent $[1,2]$ and that this theorem fails in the case of an infinite system. The algebraic approach to this problem was first given by Kastler [2]. We follow the same method and study the problem of equivalence in terms of states on the $C^{*}$-algebra (Weyl algebra) associated with the canonical commutation relations.

In particular we use the $C^{*}$-algebra $\overline{\Delta(H, \sigma)}$, built on a symplectic space $(H, \sigma)$, as introduced by Manuceau [3].

Quasi-free states of the canonical commutation relations were introduced by Robinson [4]. These states were intensively studied by Manuceau and Verbeure [5] who introduced their $C^{*}$-algebraic formulation. In this work we study the quasi-equivalence of such quasi-free states. Our approach is very closely related to the work of Powers and Størmer [6] on quasi-equivalence of gauge invariant quasi-free states of the canonical anticommutation relations. Together with Verbeure we proved a necessary and sufficient condition for two pure quasi-free states on the Weyl algebra to be unitarily equivalent [7]. To find a criterium in the case of more general quasi-free states, we used the idea of Powers and Størmer and reduced the latter problem to the case of pure states.

$\star$ Aspirant van het Belgisch N.F. W. O. On leave from the University of Louvain (Belgium).

13 Commun. math Phys., Vol. 21 
In Section 2 we recall the definition of the $C^{*}$-algebra $\overline{\Delta(H, \sigma)}$ and quasi-free states on this algebra. In Section 3 we extend every quasi-free state $\omega_{A}$ on $\overline{\Delta(H, \sigma)}$ to a pure quasi-free state $\omega_{E_{A}}$ on the larger algebra $\overline{\Delta(H \oplus H, \sigma \oplus-\sigma)}$. The inequality relating the norm differences of two quasi-free states and their extended pure states obtained by Powers and Størmer [6] for the Clifford algebra is the main result of Section 4. In the next section we prove some properties of operators on a symplectic space, which are used in the last section to prove the main theorem on quasi-equivalence.

We are indebted to Dr. A. Verbeure for many valuable discussions and especially for the Lemma's 3 and 5 below which are crucial in this work. We are also grateful to Prof. E. Størmer for his kind hospitality at the University of Oslo, and for helpful discussions.

\section{Quasi-Free States over the Weyl Algebra $\overline{\Delta(H, \sigma)}$}

For completeness we recall the definition of the Weyl algebra $\overline{\Delta(H, \sigma)}$.

Let $(H, \sigma)$ be a separable symplectic space, i.e. a real vector space $H$, equipped with a regular, antisymmetric, real bilinear form. Hence $H$ is a locally convex topological space equipped with the topology defined by the semi-norms,

$$
\varrho_{\varphi}: \psi \rightarrow|\sigma(\varphi, \psi)|
$$

and we suppose that $H$ is complete for this topology, we call $H \sigma$-complete.

Let $\Delta(H, \sigma)$ be the algebra generated by finite linear combinations of the functionals $\delta: \psi \in H \rightarrow \delta_{\psi}$ defined by

$$
\begin{aligned}
& \delta_{\psi}(\varphi)=0 \quad \text { if } \quad \psi \neq \varphi \\
& =1 \quad \text { if } \quad \psi=\varphi
\end{aligned}
$$

with the product law:

$$
\delta_{\psi} \delta_{\varphi}=e^{-i \sigma(\psi, \varphi)} \delta_{\psi+\varphi} .
$$

The mapping $\delta_{\psi} \rightarrow \delta_{\psi}^{*}=\delta_{-\psi}$ is an involution and

$$
\left\|\sum_{i=1}^{n} a_{i} \delta_{\psi_{i}}\right\|_{1}=\sum_{i=1}^{n}\left|a_{i}\right| \quad \text { with } \quad a_{i} \in \mathbb{C}
$$

is a norm on $\Delta(H, \sigma)$ such that $\Delta(H, \sigma)$ turns out to be a normed *-algebra.

The set of representations $\pi$ of $\Delta(H, \sigma)$ such that the mapping $\lambda \in \mathbb{R} \rightarrow \pi\left(\delta_{\lambda \psi}\right)$ is strongly continuous, determines a unique $C^{*}$-algebra norm on $\Delta(H, \sigma)$.

Its closure $\mathscr{A}=\overline{\Delta(H, \sigma)}$ is a $C^{*}$-algebra, which we call the Weyl algebra. For more details see [3]. 
A state on the Weyl algebra is a positive linear functional, normalized to one.

We consider the set $S$ of linear operators $A$ defined every-where on $H$, satisfying

i) $s_{A}(\psi, \varphi) \equiv-\sigma(A \psi, \varphi)$ is a real scalar product on $H$,

ii) $A^{*} A \geqq 1$ with respect to $s_{A}$.

It follows from condition i) that the adjoint $A^{*}$ of $A$ with respect to $S_{A}$ and the adjoint $A^{+}$of $A$ with respect to $\sigma$ satisfy $A^{+}=A^{*}=-A$. From condition ii) we can deduce that $A$ is invertible. Clearly $A$ is injective because $A^{*} A \geqq \mathbf{1}$ and $A$ is surjective because for any $\psi \in H$ orthogonal to the range $\mathscr{R}(A)$ of $A$ and for all $\varphi \in H$ we have $s_{A}(\psi, A \varphi)=0$. From $A^{*}=-A$ and the definition of $s_{A}$ it follows that $\sigma\left(A^{2} \psi, \varphi\right)=0$ for all $\varphi \in H$ and by the regularity of $\sigma$ that $-A^{2} \psi=A^{*} A \psi=\mathbf{0}$. So by ii) $\psi=\mathbf{0}$ and $\mathscr{R}(A)=H$.

On the other hand Schwartz inequality implies

and so

$$
\forall \psi, \varphi \in H:\left|s_{A}\left(A^{-1} \psi, \varphi\right)\right|^{2} \leqq s_{A}\left(A^{-1} \psi, A^{-1} \psi\right) s_{A}(\varphi, \varphi)
$$

because

$$
|\sigma(\psi, \varphi)|^{2} \leqq s_{A}(\psi, \psi) s_{A}(\varphi, \varphi)
$$

$$
A^{*} A \geqq \mathbf{1} \text { and } s_{A}=-\sigma \circ A .
$$

It follows from this last inequality and the work of Manuceau and Verbeure [5], that the linear functional on $\Delta(H, \sigma)$ defined by

$$
\omega_{A}\left(\delta_{\psi}\right)=\exp \left[-\frac{1}{2} s_{A}(\psi, \psi)\right]
$$

extends to a quasi-free state on $\overline{\Delta(H, \sigma)}$.

The aim of this work is to study the quasi-equivalence of any two such quasi-free states $\omega_{A}$ and $\omega_{B}$ with $A, B \in S$. We follow the method of Powers and Størmer in their study of quasi-free states on the Clifford algebra, i.e. we construct with any quasi-free state $\omega_{A}$ on $\overline{\Delta(H, \sigma)}$ a pure quasi-free state $\omega_{E_{A}}$ on $\overline{\Delta(H \oplus H, \sigma \oplus-\sigma)}$ and we prove that two states $\omega_{A}$ and $\omega_{B}$ are quasi-equivalent iff the corresponding pure states $\omega_{E_{A}}$ and $\omega_{E_{B}}$ are unitarily equivalent. In a previous paper [7], we found a necessary and suffivient condition for two pure states to be unitarily equivalent, yielding a condition in terms of $E_{\mathrm{A}}$ and $E_{\mathrm{B}}$. Finally we rewrite this criterium in terms of $A$ and $B$.

We start with the construction of the pure state $\omega_{E_{A}}$.

\section{Construction of the Pure Quasi-Free State $\omega_{E_{A}}$ Associated with $\omega_{A}$}

The symplectic form $\sigma$ on $H$ induces a new symplectic form, denoted by $\sigma \oplus-\sigma$, on $H \oplus H$ defined by

$$
(\sigma \oplus-\sigma)\left(\psi_{1} \oplus \varphi_{1}, \psi_{2} \oplus \varphi_{2}\right)=\sigma\left(\psi_{1}, \psi_{2}\right)-\sigma\left(\varphi_{1}, \varphi_{2}\right) .
$$


If $\sigma$ is regular, then so is $\sigma \oplus-\sigma$; if $H$ is $\sigma$-complete then $H \oplus H$ is $(\sigma \oplus-\sigma)$-complete.

Any operator $A$ in $S$ is invertible and from $A^{*} A=-A^{2} \geqq \mathbf{1}$ it follows that $1+A^{-2}$ is positive and hermitian (with respect to $s_{A}$ ) and has a unique square root $\sqrt{1+A^{-2}}$ which is also positive and hermitian. We then define the operator $E_{A}$ on $H \oplus H$ by

$$
E_{A}=\left(\begin{array}{cc}
A & A \sqrt{\mathbf{1}+A^{-2}} \\
-A \sqrt{\mathbf{1}+A^{-2}} & -A
\end{array}\right) .
$$

This operator $E_{A}$ satisfies conditions i) and ii) of Section 2. To prove i) we evaluate $S_{E_{A}}\left(\psi_{1} \oplus \varphi_{1}, \psi_{2} \oplus \varphi_{2}\right)$

$$
\begin{aligned}
& s_{E_{A}}\left(\psi_{1} \oplus \varphi_{1}, \psi_{2} \oplus \varphi_{2}\right)=-\sigma\left(A \psi_{1}, \psi_{2}\right)-\sigma\left(A \varphi_{1}, \varphi_{2}\right) \\
&-\sigma\left(A \sqrt{\mathbf{1}+A^{-2}} \psi_{1}, \varphi_{2}\right)-\sigma\left(A \sqrt{\mathbf{1 + A ^ { - 2 }}} \varphi_{1}, \psi_{2}\right) \\
&=s_{A}\left(\psi_{1}, \psi_{2}\right)+s_{A}\left(\varphi_{1}, \varphi_{2}\right)+s_{A}\left(\sqrt{\mathbf{1}+A^{-2}} \psi_{1}, \varphi_{2}\right)+s_{A}\left(\sqrt{\mathbf{1}+A^{-2}} \varphi_{1}, \psi_{2}\right) .
\end{aligned}
$$

Clearly $s_{E_{A}}$ is bilinear and symmetric since $s_{A}$ is a scalar product and $\sqrt{\mathbf{1}+A^{-2}}$ is hermitian. Moreover, as $-A^{2} \geqq \mathbf{0}$ and so $\mathbf{1}+A^{-2} \leqq \mathbf{1}$ we find that

$$
s_{A}(\varphi, \varphi) \geqq s_{A}\left(\left(\mathbf{1}+A^{-2}\right) \varphi, \varphi\right)=s_{A}\left(\sqrt{\mathbf{1}+A^{-2}} \varphi, \sqrt{\mathbf{1}+A^{2}} \varphi\right) .
$$

It follows that

$$
\begin{aligned}
& s_{E_{A}}(\psi \oplus \varphi, \psi \oplus \varphi) \geqq s_{A}(\psi, \psi)+s_{A}\left(\sqrt{\mathbf{1 + A ^ { - 2 }}} \varphi, \sqrt{\mathbf{1 + A ^ { - 2 }}} \varphi\right) \\
&+s_{A}\left(\sqrt{\mathbf{1 + A ^ { - 2 }}} \psi, \varphi\right)+s_{A}\left(\varphi, \sqrt{\mathbf{1 + A ^ { - 2 }}} \psi\right) \\
&=s_{A}\left(\psi+\sqrt{\mathbf{1 + A ^ { - 2 }}} \varphi, \psi+\sqrt{\mathbf{1 + A ^ { - 2 }}} \varphi\right)
\end{aligned}
$$

and that $s_{E_{A}}$ is positive because $s_{A}$ is a scalar product. On the other hand $s_{E_{A}}(\psi \oplus \varphi, \psi \oplus \varphi)=0$ implies $\psi=-\sqrt{\mathbf{1}+A^{-2}} \varphi=\left(\mathbf{1}+A^{-2}\right) \psi$ and so that $A^{2} \psi=\left(A^{2}+1\right) \psi$. It follows that $\psi$ and hence $\varphi$ and $\psi \oplus \varphi$ is zero.

To prove ii) we just compute $E_{A}^{*} E_{A}=-E_{A}^{2}$ and we find $-E_{A}^{2}=\mathbf{1}$.

Therefore $\omega_{E_{A}}$ is not only a quasi-free state on $\overline{(H \oplus H, \sigma \oplus-\sigma)}$ but even a pure quasi-free state [5].

Note that the choice of $\sigma \oplus-\sigma$ as symplectic form on $H \oplus H$ instead of $\sigma \oplus \sigma$ is crucial because if the latter was chosen, it would be impossible to find a $E_{A}$ such that $\omega_{E_{A}}$ is pure.

The pure state $\omega_{E_{A}}$ corresponds with the one of Powers and Størmer. They prove that $\omega_{E_{A}}$ and $\omega_{E_{B}}$ are uniterily equivalent iff $\omega_{A}$ and $\omega_{B}$ are quasi-equivalent. The main difficulty lies in the proof of the inequality

$$
\frac{1}{4}\left\|\omega_{E_{A}}-\omega_{E_{B}}\right\|^{2} \leqq\left\|\omega_{A}-\omega_{B}\right\| \leqq\left\|\omega_{E_{A}}-\omega_{E_{B}}\right\| .
$$


They use the fact that $\omega_{E_{A}}$ can be obtained by a "purification map" $\Phi$ from the state $\omega_{A}$ in the case where the underlying Hilbert space is finite dimensional. The infinite dimensional case is handled by continuity. We proceed in the same manner and first prove the inequality in the finite dimensional case.

\section{Locally Normal States $\overline{\Delta(H, \sigma)}$}

It is easy to see that

$$
\delta_{\psi} \in \overline{\Delta(H, \sigma)} \rightarrow \delta_{\psi \oplus \mathbf{0}} \in \overline{\Delta(H \oplus H, \sigma \oplus-\sigma)}
$$

extends to a homomorfism and that $\overline{\Delta(H, \sigma)}$ can be imbedded as a subalgebra in $\overline{\Delta(H \oplus H, \sigma \oplus-\sigma)}$. Moreover by the fact that $E_{A}$ restricted to $H \oplus\{\boldsymbol{0}\}$ coincides with $A$ it follows that the restriction of $\omega_{E_{A}}$ to the subalgebra $\overline{\Delta(H, \sigma)}$ is equal to $\omega_{A}$. Therefore

$$
\left\|\left(\omega_{A}-\omega_{B}\right)\right\| \leqq\left\|\left(\omega_{E_{A}}-\omega_{E_{B}}\right)\right\| .
$$

The main part of this section is now devoted to the fact that $\omega_{E_{A}}$ can be obtained from $\omega_{A}$ by a purification map $\Phi$ in the sense of [6].

We show this result gradually and we suppose first that $H$ is 2dimensional. Let $J$ be an operator in $S$ which is unitary. Suppose that $\varphi \in H$ is normalised such that $s_{J}(\varphi, \varphi)=1$. With $J$ there corresponds a Fock representation $\pi_{J}$ of $\overline{\Delta(H, \sigma)}$ and the creation and annihilation operators can be defined as follows

$$
\begin{aligned}
& B^{+}=\frac{1}{2}(B(\varphi)-i B(J \varphi)), \\
& B^{-}=\frac{1}{2}(B(\varphi)+i B(J \varphi)),
\end{aligned}
$$

where $B(\psi)$ satisfies $\pi_{J}\left(\delta_{\psi}\right)=e^{i B(\psi)}$ (see Ref. [5]).

Having defined these operators we can prove the following lemma's, the first two of them being trivial and therefore mentioned without proof. We assume that $H$ is two-dimensional.

Lemma 1. If $0 \leqq y<1$ then $\sum_{n=0}^{\infty} y^{n} \frac{(k+n) !}{k ! n !}=(1-y)^{-k-1}$.

Lemma 2. If $z \in \mathbb{C}$ is such that $\psi=\operatorname{Re} z \cdot \varphi+\operatorname{Im} z \cdot J \varphi$ then

$$
\begin{aligned}
\pi_{J}\left(\delta_{\psi}\right)=e^{i B(\psi)} & =e^{z z^{*} / 2} e^{i z^{*} B^{-}} e^{i z B^{+}} \\
& =e^{-z z^{*} / 2} e^{i z B^{+}} e^{i z^{*} B^{-}} .
\end{aligned}
$$

This is an immediate result of the Baker-Campdell-Hausdorff formula and the fact that $\left[B^{-}, B^{+}\right]_{-}=\mathbf{1}$. 
Lemma 3. ( $A$. Verbeure). Let $A \in S$, as $H$ is two-dimensional, we can denote its polar decomposition as $A=J$ a where a is a positive real number. There exists a density matrix $\sigma_{A}$ in the Fock representation $\pi_{J}$ of $\overline{\Delta(H, \sigma)}$ in $\mathscr{H}_{J}$ such that $\forall x \in \overline{\Delta(H, \sigma)} \Rightarrow \omega_{A}(x)=\operatorname{Tr}_{\mathscr{H}_{J}} \sigma_{A} \pi_{J}(x)$.

Proof. The density matrix is defined by $(n: o \rightarrow \infty)$

$$
\sigma=\Sigma \sigma_{n} P_{n} \quad \text { where } \quad \sigma_{n}=\frac{2}{a+1}\left(\frac{a-1}{a+1}\right)^{n}
$$

and $P_{n}$ the orthogonal projection on the vector $f_{n}=\frac{\left(B^{+}\right)^{n}}{\sqrt{n !}} \Omega_{J}, \Omega_{J}$ the cyclic vector of $\pi_{J}$.

Since $A^{*} A \geqq \mathbf{1}$, we have $a=|A| \geqq 1$ and so $\mathbf{0} \leqq \sigma$. Moreover

$$
\operatorname{Tr} \sigma=\Sigma \sigma_{n}=\frac{2}{a+1}\left(1-\frac{a-1}{a+1}\right)^{-1}=1
$$

and $\sigma$ is indeed a density matrix. It remains to show that $\omega_{A}(x)$ $=\sum_{n} \sigma_{n}\left(f_{n}, \pi_{J}(x) f_{n}\right)$ and it is sufficient to show this for $x=\delta_{\psi}$ with $\psi$ arbitrary.

As in Lemma 2 we introduce $z \in \mathbb{C}$ such that $\psi=\operatorname{Re} z \cdot \varphi+\operatorname{Im} z \cdot J \varphi$, then it follows immediately from the definition of $\omega_{A}, J$ and $\varphi$ that

$$
\omega_{A}\left(\delta_{\psi}\right)=\exp \left[-\frac{1}{2} s_{J}(a \psi, \psi)\right]=\exp \left(-\frac{a}{2} z z^{*}\right) .
$$

On the other hand by Lemma 2 and the definition and the orthonormality of the set $\left\{f_{n}\right\}$ we find

$$
\left(f_{n}, \pi_{J}\left(\delta_{\psi}\right) f_{n}\right)=\exp \left(\frac{z z^{*}}{2}\right) \sum_{k}(-1)^{k} \frac{\left(z^{*} z\right)^{k}}{k !} \frac{(k+n) !}{k ! n !}
$$

and so

$$
\begin{aligned}
\operatorname{Tr}_{\mathscr{H}_{J}} \sigma_{A} \pi_{J}\left(\delta_{\psi}\right) & =\sum_{n} \sigma_{n}\left(f_{n}, \pi_{J}\left(\delta_{\psi}\right) f_{n}\right) \\
& =\sum_{k} \exp \left(\frac{z z^{*}}{2}\right)(-1)^{k} \frac{\left(z^{*} z\right)^{k}}{k !} \frac{2}{a+1} \sum_{n}\left(\frac{a-1}{a+1}\right)^{n} \frac{(k+n) !}{k ! n !} .
\end{aligned}
$$

Using Lemma 1 we can evaluate the last sum and we find easily

$$
e^{z z^{*} / 2} \exp \left(-\frac{a+1}{2} z z^{*}\right)=\exp \left(-\frac{a}{2} z z^{*}\right)
$$

so that

$$
\operatorname{Tr}_{\mathscr{C}_{J}} \sigma_{A} \pi_{J}\left(\delta_{\psi}\right)=\omega_{A}\left(\delta_{\psi}\right) \text {. Q.E.D. }
$$


Lemma 4. Let $\omega_{A}$ be a quasi-free state on $\overline{\Delta(H, \sigma)}, H$ two-dimensional. The pure state $\omega_{E_{A}}$ on $\overline{\Delta(H \oplus H, \sigma \oplus-\sigma)}$ can be obtained by a purification map $\Phi$ from $\omega_{A}$ (in the sense of [6]).

Proof. Powers and Størmer constructed this purification map from a normal state $\omega$ on a finite irreducible matrix algebra $M$ to a pure state $\Phi(\omega)$ on the algebra $M \otimes M$.

We consider the Fock representation $\pi_{J}$ of $\overline{\Delta(H, \sigma)}$ and we set $M=\pi_{J} \overline{(\Delta(H, \sigma))}$. The state $\omega_{A}$ induces a normal state $\omega(Q)=\operatorname{Tr} \sigma_{A} Q$ on $M$ by Lemma 3. If $V$ is any anti-unitary operator on $\mathscr{H}_{J}$, we can extend $\omega$ to a pure state $\Phi(\omega)$ on $M \otimes M$, defined by (see Ref. [6])

$$
\Phi(\omega)(P \otimes Q)=\sum_{n, m} \sqrt{\sigma_{n}} \sqrt{\sigma_{m}^{-}}\left(f_{n}, P f_{m}\right)\left(V f_{n}, Q V f_{m}\right) .
$$

We now define the state $\Phi\left(\omega_{A}\right)$ on the algebra $\overline{\Delta(H \oplus H, \sigma \oplus-\sigma)}$ by

$$
\Phi\left(\omega_{A}\right)\left(\delta_{\psi_{1} \oplus \psi_{2}}\right)=\Phi(\omega)\left(\pi_{J}\left(\delta_{\psi_{1}}\right) \otimes V \pi_{J}\left(\delta_{-\psi_{2}}\right) V^{-1}\right) .
$$

We proceed by showing that $\Phi\left(\omega_{A}\right)=\omega_{E_{A}}$. Clearly

$$
\Phi\left(\omega_{A}\right)\left(\delta_{\psi_{1} \oplus \psi_{2}}\right)=\sum_{n, m} \sqrt{\sigma_{n}} \sqrt{\sigma_{m}}\left(f_{n}, \pi_{J}\left(\delta_{\psi_{1}}\right) f_{m}\right)\left(V f_{n}, V \pi_{J}\left(\delta_{-\psi_{2}}\right) f_{m}\right)
$$

and as $V$ is anti-unitary and $\pi_{J}\left(\delta_{-\psi}\right)=\pi_{J}\left(\delta_{\psi}\right)^{*}$

$$
\Phi\left(\omega_{A}\right)\left(\delta_{\psi_{1} \oplus \psi_{2}}\right)=\sum_{n, m} \sqrt{\sigma_{n}} \sqrt{\sigma_{m}}\left(f_{n}, \pi_{J}\left(\delta_{\psi_{1}}\right) f_{m}\right)\left(f_{m}, \pi_{J}\left(\delta_{\psi_{2}}\right) f_{n}\right)
$$

so that $\Phi\left(\omega_{A}\right)$ is independent of the choice of $V$.

We can define the complex numbers $z$ and $y$ so that

$$
\psi_{1}=\operatorname{Re} z \cdot \varphi+\operatorname{Im} z \cdot J \varphi, \quad \psi_{2}=\operatorname{Re} y \cdot \varphi+\operatorname{Im} y \cdot J \varphi
$$

and by Lemma 2 we find

$$
\begin{aligned}
& \Phi\left(\omega_{A}\right)\left(\delta_{\psi_{1} \oplus \psi_{2}}\right)=\sum_{n, m} \sqrt{\sigma_{n}} \sqrt{\sigma_{m}} e^{-z z^{*} / 2} e^{y y^{*} / 2}\left(f_{n}, e^{i z B^{+}} e^{i z^{*} B^{-}} f_{m}\right) \\
& \cdot\left(f_{m}, e^{i y^{*} B^{-}} e^{i y B^{+}} f_{n}\right) .
\end{aligned}
$$

Again by a straightforward calculation and application of the definition of the vectors $\left\{f_{n}\right\}$ and $\left[B^{-}, B^{+}\right]_{-}=\mathbf{1}$ we get

$$
\begin{aligned}
& \Phi\left(\omega_{A}\right)\left(\delta_{\psi_{1} \oplus \psi_{2}}\right)= \sum_{n, m} \sqrt{\sigma_{n}} \sqrt{\sigma_{m}} e^{-z z^{*} / 2} e^{y y^{*} / 2} \\
& \cdot \sum_{r, s} \frac{s !}{r !} \frac{\left(i y^{*}\right)^{s-m}}{(s-m) !} \frac{(i y)^{s-n}}{(s-n) !} \frac{(i z)^{n-r}}{(n-r) !} \frac{\left(i z^{*}\right)^{m-r}}{(m-r) !} \\
&=e^{-z z^{*} / 2} e^{y y^{*} / 2} \sum_{r, s} \frac{s !}{r !}(-1)^{s-r}\left|\left(\sum_{n} \sqrt{\sigma_{n}} \frac{z^{n-r}}{(n-r) !} \frac{y^{s-n}}{(s-n) !}\right)\right|^{2} .
\end{aligned}
$$


For sake of simplicity we introduce $c=\frac{a+1}{2}$ so that $\sigma_{n}=\frac{1}{c}\left(1-\frac{1}{c}\right)^{n}$. Then by the use of the formula

$$
\begin{aligned}
\sum_{n} \sqrt{\sigma_{n}} \frac{z^{n-r}}{(n-r) !} \frac{y^{s-n}}{(s-n) !} & =\sum \frac{1}{\sqrt{c}} \frac{(1-c)^{r / 2}}{c^{s / 2}} \frac{(\sqrt{c-1} z)^{n-r}}{(n-r) !} \frac{(\sqrt{c} y)^{s-n}}{(s-n) !} \\
& =\frac{1}{\sqrt{c}} \frac{(1-c)^{r / 2}}{c^{s / 2}} \frac{(\sqrt{c-1} z+\sqrt{c} y)^{s-r}}{(s-r) !}
\end{aligned}
$$

and the substitutions $t=s-r$ and $x=\sqrt{c-1} z+\sqrt{c} y$ we get

$$
\begin{aligned}
\Phi\left(\omega_{A}\right)\left(\delta_{\psi_{1} \oplus \psi_{2}}\right) & =e^{-z z^{*} / 2} e^{y y^{*} / 2} \sum_{t}(-1)^{t} \frac{\left(x x^{*}\right)^{t}}{t !} \sum_{r} \frac{(t+r) !}{r ! t !}\left(1-\frac{1}{c}\right)^{r} \frac{1}{c^{t+1}} \\
& =e^{-z z^{*} / 2} e^{y y^{*} / 2} e^{-x x^{*}}
\end{aligned}
$$

by Lemma 1 .

On the other hand by the definition of $E_{A}$ we find

$$
\omega_{E_{A}}\left(\delta_{\psi_{1} \oplus \psi_{2}}\right)=\exp \left(\frac{1}{2} \sigma\left(A \psi_{1}, \psi_{1}\right)+\frac{1}{2} \sigma\left(A \psi_{2}, \psi_{2}\right)+\sigma\left(A \sqrt{\mathbf{1}+A^{-2}} \psi_{1}, \psi_{2}\right)\right) \text {. }
$$

Furthermore we have $A=J a$ and so

$$
\sigma\left(A \sqrt{\mathbf{1}+A^{-2}} \psi_{1}, \psi_{2}\right)=\sqrt{a^{2}-1} \sigma\left(J \psi_{1}, \psi_{2}\right) .
$$

Introducing again the complex numbers $z$ and $y$ we find

$$
\omega_{E_{A}}\left(\delta_{\psi_{1} \oplus \psi_{2}}\right)=\exp \left(-\frac{a}{2} z z^{*}-\frac{a}{2} y y^{*}-\frac{\sqrt{a^{2}-1}}{2}\left(y z^{*}+z y^{*}\right)\right)
$$

and with the definitions of $c$ and $x$ it is easy to verify that $\omega_{E_{A}}\left(\delta_{\psi_{1} \oplus \psi_{2}}\right)$ $=\Phi\left(\omega_{A}\right)\left(\delta_{\psi_{1} \oplus \psi_{2}}\right)$. Q.E.D.

We next prove Lemma 3 and 4 in the case that $H$ is of finite dimension $2 k$ using the fact that the considered quasi-free states are product states.

Lemma 5. ( $A$. Verbeure.) Let $H$ be $2 k$-dimensional and denote by $A=J|A|$ the polar decomposition of $A \in S$. There exists a density matrix $\sigma_{A}$ in the Fock representation $\pi_{J}$ of $\overline{\Delta(H, \sigma)}$ in $\mathscr{H}_{J}$ such that

$$
\forall x \in \overline{\Delta(H, \sigma)} \Rightarrow \omega_{A}(x)=\operatorname{Tr}_{\mathscr{H}_{J}} \sigma_{A} \pi_{J}(x) .
$$

Proof. The operator $|\mathrm{A}|$ can be diagonalised as $|A|=\sum_{i=1}^{k} a_{i} P_{i}$. Since $J$ commutes with $|A|$, we can find a symplectic basis $\left\{\varphi_{i}, J \varphi_{i}\right\}$ with $\sigma\left(\varphi_{i}, J \varphi_{j}\right)=\delta_{i j}, \sigma\left(\varphi_{i}, \varphi_{j}\right)=\sigma\left(J \varphi_{i}, J \varphi_{j}\right)=0$ and $|A| \varphi_{i}=a_{i} \varphi_{i},|A| J \varphi_{i}=a_{i} J \varphi_{i}$.

With every pair $\left(\varphi_{i}, J \varphi_{i}\right)$ we associate the creation operator

$$
B_{i}^{+}=\frac{1}{2}\left(B\left(\varphi_{i}\right)-i B\left(J \varphi_{i}\right)\right)
$$


and for every set of $k$ non-negative integers $(n)=\left\{n_{1}, n_{2} \ldots n_{k}\right\}$ we define

and

$$
f_{(n)}=\left[\prod_{i=1}^{k}\left(\frac{\left(B_{i}^{+}\right)^{n_{i}}}{\sqrt{n_{i} !}}\right)\right] \Omega_{J}
$$

$$
\sigma_{(n)}=\prod_{i=1}^{k}\left(\frac{1}{c_{i}}\left(1-\frac{1}{c_{i}}\right)^{n_{i}}\right) \quad c_{i}=\frac{a_{i}+1}{2} .
$$

By a straightforward extension of the results of Lemma 3 one can prove that $\sigma_{A}=\sum_{(n)} \sigma_{(n)} P_{f_{(n)}}$ is a density matrix and that

$$
\omega_{A}\left(\delta_{\psi}\right)=\operatorname{Tr}_{\mathscr{H}_{J}} \sigma_{A} \pi_{J}\left(\delta_{\psi}\right) \quad \text { for every } \quad \psi \in H .
$$

Lemma 6. Let $\omega_{A}$ be a quasi-free state on $\overline{\Delta(H, \sigma)}$ with $H$ finitedimensional. Given an arbitrary Fock representation $\pi_{K}$ of $\overline{\Delta(H, \sigma)}$ there exists a density matrix $\sigma_{A}(K)$ such that

$$
\forall x \in \overline{\Delta(H, \sigma)} \Rightarrow \omega_{A}(x)=\operatorname{Tr}_{\mathscr{H}_{K}} \sigma_{A}(K) \pi_{K}(x) .
$$

Proof. This results immediately from the unitary equivalence of the Fock representations of $\overline{\Delta(H, \sigma)}$ if $H$ is finite-dimensional $[1,2]$.

The density matrix $\sigma_{A}(K)$ is given by

$$
\sigma_{A}(K)=\sum_{(n)} \sigma_{(n)} P_{g_{(n)}}
$$

with $g_{(n)}=U_{K J} f_{(n)}$ and $U_{K J}$ the unitary operator of $\mathscr{H}_{J} \rightarrow \mathscr{H}_{K}$. In the next lemma we prove an estimate for the norm differences $\left\|\omega_{E_{A}}-\omega_{E_{B}}\right\|$ and $\left\|\omega_{A}-\omega_{B}\right\|$ in the finite dimensional case. Note that this inequality is precisely the same as the one obtained by Powers and Størmer for the Clifford algebra.

Lemma 7. If $\omega_{A}$ and $\omega_{B}$ are two quasi-free states on $\overline{\Delta(H, \sigma)}$ with $H$ finite-dimensional, $\omega_{E_{A}}$ and $\omega_{E_{B}}$ the extended pure states on $\overline{\Delta(H \oplus H, \sigma \oplus-\sigma)}$ then

$$
\frac{1}{4}\left\|\omega_{E_{A}}-\omega_{E_{B}}\right\|^{2} \leqq\left\|\omega_{A}-\omega_{B}\right\| .
$$

Proof. We consider an arbitrary Fock representation $\pi_{K}$ and we define the normal states $\omega_{1}$ and $\omega_{2}$ on $M=\pi_{K} \overline{(\Delta(H, \sigma))}$ by

$$
\omega_{1}=\operatorname{Tr}_{\mathscr{H}_{K}} \sigma_{A}(K) . \quad \omega_{2}=\operatorname{Tr}_{\mathscr{H}_{K}} \sigma_{B}(K) .
$$

For the norm difference we find

$$
\left\|\omega_{1}-\omega_{2}\right\|=\sup _{\substack{\|P\| \leq 1 \\ P \in \bar{M}}}\left|\omega_{1}(P)-\omega_{2}(P)\right|=\sup _{\substack{\left\|\pi_{K}(x)\right\| \leq 1 \\ x \in \Delta(H, \sigma)}}\left|\omega_{1}\left(\pi_{K}(x)\right)-\omega_{2}\left(\pi_{K}(x)\right)\right| .
$$


Since $\Delta(H, \sigma)$ is dense in $\overline{\Delta(H, \sigma)}$ and $\left\|\pi_{K}(x)\right\|=\|x\|$ if $x \in \Delta(H, \sigma)$ [3] we get

$$
\left\|\omega_{1}-\omega_{2}\right\|=\sup _{\substack{\|x\| \leq 1 \\ x \in \Delta(\bar{H}, \sigma)}}\left|\omega_{A}(x)-\omega_{B}(x)\right|=\left\|\omega_{A}-\omega_{B}\right\| .
$$

Given an anti-unitary operator $V$ we again construct the states $\Phi\left(\omega_{1}\right)$ and $\Phi\left(\omega_{2}\right)$ by

$$
\Phi(\omega)(P \otimes Q)=\sum_{(n)(m)} \sqrt{\sigma_{(n)}} \sqrt{\sigma_{(m)}}\left(g_{(n)}, P g_{(m)}\right)\left(V g_{(n)}, Q V g_{(m)}\right) .
$$

Powers and Størmer [6] proved the inequality $\frac{1}{4}\left\|\Phi\left(\omega_{1}\right)-\Phi\left(\omega_{2}\right)\right\|^{2}$ $\leqq\left\|\omega_{1}-\omega_{2}\right\|$ in the case of matrix algebras, but it is not hard to extend the result to the case of normal states on irreducible operator algebras [8].

Next we define the states $\Phi\left(\omega_{A}\right)$ and $\Phi\left(\omega_{B}\right)$ on $\Delta(H \oplus H, \sigma \oplus-\sigma)$ by

$$
\Phi\left(\omega_{A}\right)\left(\delta_{\psi_{1} \oplus \psi_{2}}\right)=\Phi\left(\omega_{1}\right)\left(\pi_{K}\left(\delta_{\psi_{1}}\right) \otimes V \pi_{K}\left(\delta_{-\psi_{2}}\right) V^{-1}\right) .
$$

From the fact that $\pi_{K}$ is a representation of $\overline{\Delta(H, \sigma)}$ it can be shown in a straightforward manner that

$$
\xi=\pi_{K} \otimes V \pi_{K}^{*} V^{-1}
$$

is a representation of $\overline{\Delta(H \oplus H, \sigma \oplus-\sigma)}$ in $M \otimes M$ and it follows that $(x \in \overline{\Delta(H \oplus H, \sigma \oplus-\sigma)})$

$$
\begin{aligned}
\left\|\Phi\left(\omega_{A}\right)-\Phi\left(\omega_{B}\right)\right\| & =\sup _{\|x\| \leqq 1}\left|\Phi\left(\omega_{A}\right)(x)-\Phi\left(\omega_{B}\right)(x)\right| \\
& =\sup _{\|x\| \leqq 1}\left|\Phi\left(\omega_{1}\right)(\xi(x))-\Phi\left(\omega_{2}\right)(\xi(x))\right| \\
& \leqq \sup _{\|\xi(x)\| \leqq 1}\left|\Phi\left(\omega_{1}\right)(\xi(x))-\Phi\left(\omega_{2}\right)(\xi(x))\right|
\end{aligned}
$$

since $\|\xi(x)\| \leqq\|x\|$.

The last expression is certainly smaller than $\left\|\Phi\left(\omega_{1}\right)-\Phi\left(\omega_{2}\right)\right\|$ so that

$$
\left\|\Phi\left(\omega_{A}\right)-\Phi\left(\omega_{B}\right)\right\| \leqq\left\|\Phi\left(\omega_{1}\right)-\Phi\left(\omega_{2}\right)\right\| .
$$

It remains to show that $\Phi\left(\omega_{A}\right)=\omega_{E_{A}}$ and $\Phi\left(\omega_{B}\right)=\omega_{E_{B}}$. From the definition of $\Phi\left(\omega_{A}\right)$ we have

$$
\Phi\left(\omega_{A}\right)\left(\delta_{\psi_{1} \oplus \psi_{2}}\right)=\sum_{(n)(m)} \sqrt{\sigma_{(n)}} \sqrt{\sigma_{(m)}}\left(g_{(n)}, \pi_{K}\left(\delta_{\psi_{1}}\right) g_{(m)}\right)\left(g_{(m)}, \pi_{K}\left(\delta_{\psi_{2}}\right) g_{(n)}\right)
$$

and using the unitary equivalence of Fock representations

$$
\Phi\left(\omega_{A}\right)\left(\delta_{\psi_{1} \oplus \psi_{2}}\right)=\sum_{(n)(m)} \sqrt{\sigma_{(n)}} \sqrt{\sigma_{(m)}}\left(f_{(n)}, \pi_{J}\left(\delta_{\psi_{1}}\right) f_{(m)}\right)\left(f_{(m)}, \pi_{J}\left(\delta_{\psi_{2}}\right) f_{(n)}\right)
$$


with the notations of Lemma 5. By a straightforward extension of the results of Lemma 4, it is now possible to show that again

$$
\Phi\left(\omega_{A}\right)\left(\delta_{\psi_{1} \oplus \psi_{2}}\right)=\omega_{E_{A}}\left(\delta_{\psi_{1} \oplus \psi_{2}}\right) .
$$

This completes the proof.

We now want to extend the result of Lemma 7 to the general case with $H$ infinite-dimensional. Powers and Størmer use therefore continuity considerations. Here this is slightly more difficult as the operators in $S$ may be unbounded. Nevertheless it is possible to prove the following lemma. From now on $H$ may be infinite dimensional.

Lemma 8. Given two quasi-free states $\omega_{A}$ and $\omega_{B}$ on $\overline{\Delta(H, \sigma)}$, an element $y=\sum_{i=1}^{n} a_{i} \delta_{\psi_{\imath} \oplus \varphi_{i}}$ in $\overline{\Delta(H \oplus H, \sigma \oplus-\sigma)}$ and $\varepsilon>0$, there exist a regular finite subspace $H_{0} \subset H$ and two quasi-free states $\omega_{A_{0}}$ and $\omega_{B_{0}}$ on $\overline{\Delta\left(H_{0}, \sigma\right)}$, such that

i) $y \in \overline{\Delta\left(H_{0} \oplus H_{0}, \sigma \oplus-\sigma\right)}$,

ii) $\omega_{A}\left|\overline{\Delta\left(H_{0}, \sigma\right)}=\omega_{A_{0}}, \omega_{B}\right| \overline{\Delta\left(H_{0}, \sigma\right)}=\omega_{B_{0}}$,

iii) $\left|\omega_{E_{A}}(y)-\omega_{E_{A_{0}}}(y)\right|<\varepsilon \quad\left|\omega_{E_{B}}(y)-\omega_{E_{B_{0}}}(y)\right|<\varepsilon$, where $\omega_{E_{A_{0}}}, \omega_{E_{B_{0}}}$ are to be understood as states on $\overline{\Delta\left(H_{0} \oplus H_{0}, \sigma \oplus-\sigma\right)}$

Proof. Let $J$ be an arbitrary unitary element in $S\left(J^{*} J=-J^{2}=1\right)$, define for every non-negative integer $m$ the subspace $H_{m}$ of $H$ spanned by

$$
\left\{A^{-k} \psi_{i}, B^{-k} \varphi_{i}, J A^{-k} \psi_{i}, J B^{-k} \varphi_{i} \text {; with } k: 0 \rightarrow m, i: 1 \rightarrow n\right\} .
$$

This subspace $H_{m}$ is finite for all finite $m$ and is regular since, given $\psi \neq 0$ in $H_{m}$, then $J \psi \in H_{m}$ and $\sigma(\psi, J \psi)=s_{J}(\psi, \psi) \neq 0$ so that the restriction of $\sigma$ to $H_{m}$ remains regular.

For all $m \geqq 0, \psi_{i}, \varphi_{i} \in H_{m}$ so that $y=\sum_{i=1}^{n} a_{i} \delta_{\psi_{i} \oplus \varphi_{i}}$ is an element of $\overline{\Delta\left(H_{m} \oplus H_{m}, \sigma \oplus-\sigma\right)}$.

Let $P_{m}$ be the orthogonal projection on $H_{m}$. The operators $A_{m}=P_{m} A P_{m}$

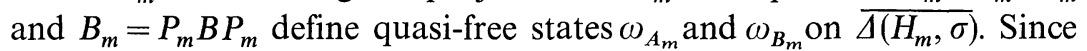
$s_{A_{m}}(\psi, \varphi)=s_{A}(\psi, \varphi)$ for $\forall \psi, \varphi \in H_{m}, s_{A_{m}}$ is a scalar product on $H_{m}$.

Moreover, for all $\psi, \varphi \in H_{m}:|\sigma(\psi, \varphi)|^{2} \leqq s_{A_{m}}(\psi, \psi) s_{A_{m}}(\varphi, \varphi)$ and replacing $\psi$ by $A_{m} \varphi$ we get

$$
s_{A_{m}}(\varphi, \varphi)^{2} \leqq s_{A_{m}}\left(A_{m} \psi, A_{m} \psi\right) s_{A_{m}}(\varphi, \varphi)
$$

so that $A_{m}^{*} A_{m} \geqq 1$ with respect to $s_{A_{m}}$.

So we may conclude that if $H_{0}=H_{m}$ for any non-negative integer $m$, then $H_{0}$ is a finite regular subspace of $H$ and $\omega_{A_{m}}$ and $\omega_{B_{m}}$ are quasifree states on $\overline{\left(H_{m}, \sigma\right)}$ such that conditions i) and ii) of the lemma are fulfilled. If we can prove that $\lim _{m \rightarrow \infty} \omega_{E_{A_{m}}}(y)=\omega_{E_{A}}(y)$, then the lemma 
will be proved since the problem is symmetric in $A$ and $B$.

$$
\begin{aligned}
\text { Now, as } y & =\sum_{i=1}^{n} a_{i} \delta_{\psi_{i} \oplus \varphi_{i}} \text { and } \\
\omega_{E_{A_{m}}}\left(\delta_{\psi_{i} \oplus \varphi_{i}}\right) & =\exp \left[-\frac{1}{2} s_{A}\left(\psi_{i}, \psi_{i}\right)-\frac{1}{2} s_{A}\left(\varphi_{i}, \varphi_{i}\right)-s_{A}\left(\sqrt{1+A_{m}^{-2}} \psi_{i}, \varphi_{i}\right)\right]
\end{aligned}
$$

it will be sufficient that for any $\psi_{i}, \varphi_{i}(i=1, n)$

$$
\lim _{m \rightarrow \infty} s_{A}\left(\sqrt{1+A_{m}^{-2}} \psi_{i}, \varphi_{i}\right)=s_{A}\left(\sqrt{1+A^{-2}} \psi_{i}, \varphi_{i}\right)
$$

and by Schwartz inequality that

$$
\lim _{m \rightarrow \infty}\left\|\left(\sqrt{1+A_{m}^{-2}}-\sqrt{1+A^{-2}}\right) \psi_{i}\right\|_{s_{A}}=0 .
$$

To show this we consider the power series of $(1-x)^{\frac{1}{2}}=\sum_{i=0}^{\infty} c_{i} x^{i}$ converging for $0 \leqq x \leqq 1$ (This follows straightforward by usual convergence criteria), and we define

$$
g_{p}\left(A_{m}\right)=\sum_{i=0}^{p} c_{i}\left(-A_{m}^{-2}\right)^{i} .
$$

As $0 \leqq-A_{m}^{-2} \leqq 1, g_{p}\left(A_{m}\right)$ converges in norm, and hence strongly to $\sqrt{\mathbf{1}+A_{m}^{-2}}$. Similarly $g_{p}(A)$ converges strongly to $\sqrt{\mathbf{1 + A ^ { - 2 }}}$.

Therefore for any $\varepsilon>0$ there is a $p_{0}$ such that

$$
\begin{aligned}
& \left\|\left(\sqrt{\mathbf{1}+A_{m}^{-2}}-\sqrt{\mathbf{1}+A^{-2}}\right) \psi_{i}\right\|_{s_{A}} \leqq\left\|\left(\sqrt{\mathbf{1}+A_{m}^{-2}}-g_{p_{0}}\left(A_{m}\right)\right) \psi_{i}\right\|_{s_{A}} \\
& \quad+\left\|\left(\sqrt{\mathbf{1}+A^{-2}}-g_{p_{0}}(A)\right) \psi_{i}\right\|_{s_{A}}+\left\|\left(g_{p_{0}}(A)-g_{p_{0}}\left(A_{m}\right)\right) \psi_{i}\right\|_{s_{A}} \\
& \leqq \\
& \quad \varepsilon / 2+\varepsilon / 2+\left\|\left(g_{p_{0}}(A)-g_{p_{0}}\left(A_{m}\right)\right) \psi_{i}\right\|_{s_{A}}
\end{aligned}
$$

It is easy to verify that

$$
A_{m}^{-k}-A^{-k}=\sum_{j=0}^{k-1} A_{m}^{-k+j}\left(A-A_{m}\right) A^{-j-1}
$$

and $\left(A-A_{m}\right) A^{-j-1} \psi_{i}=A^{-j} \psi_{i}-P_{m} A P_{m} A^{-j-1} \psi_{i}$. If $m \geqq j+1$ then $A^{-j-1} \psi_{i} \in H_{m}$ and $A^{-j} \psi_{i} \in H_{m}$ so that $\left(A-A_{m}\right) A^{-j-1} \psi_{i}=\mathbf{0}$. So if $m \geqq k$ then $\left(A_{m}^{-k}-A^{-k}\right) \psi_{i}=\mathbf{0}$ and if $m \geqq 2 p_{0}: g_{p_{0}}\left(A_{m}\right) \psi_{i}=g_{p_{0}}(A) \psi_{i}$ so that $\left\|\left(\sqrt{\mathbf{1}+A_{m}^{-2}}-\sqrt{\mathbf{1 + A ^ { - 2 }}}\right) \psi_{i}\right\| \leqq \varepsilon$.

This completes the proof.

Lemma 9. Let $\omega_{A}$ and $\omega_{B}$ be quasi-free states on $\overline{\Delta(H, \sigma)}$ with $H$ infinite dimensional, $\omega_{E_{A}}$ and $\omega_{E_{B}}$ the extended pure states on $\overline{\Delta(H \oplus H, \sigma \oplus-\sigma)}$ then

$$
\frac{1}{4}\left\|\omega_{E_{A}}-\omega_{E_{B}}\right\|^{2} \leqq\left\|\omega_{A}-\omega_{B}\right\|
$$


Proof. Since $\Delta(H \oplus H, \sigma \oplus-\sigma)$ is dense in $\overline{\Delta(H \oplus H, \sigma \oplus-\sigma)}$, it follows that for every $\varepsilon>0$ there exists an $y$ of the form $y=\sum_{i=1}^{n} a_{i} \delta_{\psi_{i} \oplus \varphi_{i}}$ such that

$$
\|y\| \leqq 1 \quad \text { and } \quad\left\|\omega_{E_{A}}-\omega_{E_{B}}\right\|-\varepsilon<\left|\omega_{E_{A}}(y)-\omega_{E_{B}}(y)\right|
$$

By Lemma 8 there exists a subalgebra $\overline{\Delta\left(H_{0}, \sigma\right)}$ on a finite subspace $H_{0}$ and two quasi-free states $\omega_{A_{0}}$ and $\omega_{B_{0}}$ on this subalgebra such that

$$
\left|\omega_{E_{A}}(y)-\omega_{E_{B}}(y)\right| \leqq\left|\omega_{E_{A_{0}}}(y)-\omega_{E_{B_{0}}}(y)\right|+2 \varepsilon
$$

and $\omega_{A} \mid \overline{\Delta\left(H_{0}, \sigma\right)}=\omega_{A_{0}}$ and $\omega_{B} \mid \overline{\Delta\left(H_{0}, \sigma\right)}=\omega_{B_{0}}$.

So we have by Lemma 7

$$
\left|\omega_{E_{A_{0}}}(y)-\omega_{E_{B_{0}}}(y)\right| \leqq\left\|\omega_{E_{A_{0}}}-\omega_{E_{B_{0}}}\right\| \leqq 2\left\|\omega_{A_{0}}-\omega_{B_{0}}\right\|^{\frac{1}{2}}
$$

and $\left\|\omega_{A_{0}}-\omega_{B_{0}}\right\|=\left\|\left(\omega_{A}-\omega_{B}\right) \mid \overline{\Delta\left(H_{0}, \sigma\right)}\right\| \leqq\left\|\omega_{A}-\omega_{B}\right\|$. The result is $\left\|\omega_{E_{A}}-\omega_{E_{B}}\right\| \leqq 2\left\|\omega_{A}-\omega_{B}\right\|^{\frac{1}{2}}+3 \varepsilon$ and since this holds for every $\varepsilon$, we proved the lemma.

\section{Operators on a Symplectic Space}

The estimate obtained in the previous lemma is going to be used in the last section where we prove that two quasi-free factor states $\omega_{A}$ and $\omega_{B}$ are quasi-equivalent iff the corresponding pure states $\omega_{E_{A}}$ and $\omega_{E_{B}}$ are unitarily equivalent. On the other hand that is equivalent to saying that $\left(E_{A}-E_{B}\right)^{2}$ is of trace class with respect to the scalar product $s_{E_{A}}$ on $H \oplus H[7]$. This yields a necessary and sufficient condition for the quasi-equivalence of $\omega_{A}$ and $\omega_{B}$ in terms of $E_{A}$ and $E_{B}$. In this section we try to rewrite this condition in terms of $A$ and $B$ directly. The result will be used to prove that if $A-B$ is of finite rank, then $\omega_{E_{A}}$ and $\omega_{E_{B}}$ are unitarily equivalent, a lemma which we need for the proof of the theorem of the last section. That is precisely the reason why we start for searching this equivalent conditions already in this section.

We define the operator

$R \equiv\left(E_{A}-E_{B}\right)\left(E_{A}-E_{B}\right)=-2-E_{A} E_{B}-E_{B} E_{A}=-2-E_{A} E_{B}-\left(E_{A} E_{B}\right)^{-1}$.

We recall that $-E_{A} E_{B}$ was found to be hermitian and positive with respect to $s_{E_{A}}$ because $s_{E_{A}}\left(-E_{A} E_{B} \psi, \varphi\right)=-(\sigma \oplus-\sigma)\left(E_{B} \psi, \varphi\right)=s_{E_{B}}(\psi, \varphi)$ if $\psi, \varphi \in H \oplus H$.

As $-E_{B} E_{A}=\left(-E_{A} E_{B}\right)^{-1}$ the same statement is true for $-E_{B} E_{A}$. And using the fact $x+x^{-1}-2 \geqq 0$ if $x$ is positive it follows that $R$ is hermitian and positive with respect to $s_{E_{A}}$. 
We next introduce the operator $U$ on $H \oplus H$

$$
U \equiv \frac{1}{\sqrt{2}}\left(\begin{array}{rr}
1 & 1 \\
1 & -\mathbf{1}
\end{array}\right)
$$

The adjoint of $U$ with respect to $\sigma \oplus-\sigma$ is $U^{+}=\frac{1}{\sqrt{2}}\left(\begin{array}{rr}\mathbf{1} & -\mathbf{1} \\ -\mathbf{1} & -\mathbf{1}\end{array}\right)$ and it is easily verified that $U^{2}=U^{+2}=\mathbf{1}$.

Define next the operators

$$
\begin{array}{ll}
Q_{A} \equiv A+A \sqrt{\mathbf{1 + A}} A^{-2}, & Q_{B} \equiv B+B \sqrt{\mathbf{1 + B ^ { - 2 }}}, \\
P_{A} \equiv A-A \sqrt{\mathbf{1 + A ^ { - 2 }}}, & P_{B} \equiv B-B \sqrt{\mathbf{1 + B ^ { - 2 }}} .
\end{array}
$$

It is straightforward to verify that

$$
U^{+} E_{A} U=\left(\begin{array}{cc}
Q_{A} & \mathbf{0} \\
\mathbf{0} & -P_{A}
\end{array}\right)
$$

and it follows that the bilinear form

satisfies

$$
s \equiv s_{Q_{A}} \oplus s_{P_{A}}=-(\sigma \oplus-\sigma) \circ\left(\begin{array}{cc}
Q_{A} & \mathbf{0} \\
\mathbf{0} & -P_{A}
\end{array}\right)
$$

$$
\begin{aligned}
s(\psi, \varphi) & =-(\sigma \oplus-\sigma)\left(U^{+} E_{A} U \psi, \varphi\right) \\
& =-(\sigma \oplus-\sigma)\left(E_{A} U \psi, U \varphi\right)=s_{E_{A}}(U \psi, U \varphi)
\end{aligned}
$$

and therefore is a scalar product on $H \oplus H$.

Let $\left\{\psi_{i}\right\}$ be an orthonormal basis in $H \oplus H$ with respect to $s_{E_{A}}$, then $\left\{U \psi_{i}\right\}$ is an orthonormal basis with respect to $s$. As $R$ is positive and hermitian with respect to $s_{E_{A}}$, it is of trace class iff the $\operatorname{sum} \sum_{i} s_{E_{A}}\left(\psi_{i}, R \psi_{i}\right)$ converges. On the other hand by the definition of $s$ and $U^{2}=\mathbf{1}$

$$
s_{E_{A}}(\psi, R \varphi)=s(U \psi, U R U \cdot U \varphi)
$$

And it follows that $U R U$ is positive and hermitian with respect to $s$. Moreover $\sum_{i} s_{E_{A}}\left(\psi_{i}, R \psi_{i}\right)=\sum_{i} s\left(U \psi_{i}, U R U U \psi_{i}\right)$ so that $R$ is of trace class with respect to $S_{E_{A}}$ if and only if $U R U$ is of trace class with respect to $s$.

In what follows we denote by $\tau c(s)$ and $\sigma c(s)$ resp. the trace class and Hilbert Schmidt class of operators with respect to the scalar product s. So we showed already that $R \in \tau c\left(s_{E_{A}}\right) \Leftrightarrow U R U \in \tau c(s)$. 
The last operator is found to be

$$
\begin{aligned}
U R U & =U\left(E_{A}-E_{B}\right) U^{+} U^{+}\left(E_{A}-E_{B}\right) U \\
& =\left(\begin{array}{ll}
P_{A}-P_{B} & \mathbf{0} \\
\mathbf{0} & -Q_{A}+Q_{B}
\end{array}\right)\left(\begin{array}{lc}
Q_{A}-Q_{B} & \mathbf{0} \\
\mathbf{0} & -P_{A}+P_{B}
\end{array}\right)
\end{aligned}
$$

and as $U R U$ is positive and hermitian with respect to $s=s_{Q_{A}} \oplus s_{P_{A}}$, the condition is equivalent with:

and

$$
R_{1} \equiv\left(P_{A}-P_{B}\right)\left(Q_{A}-Q_{B}\right) \in \tau c\left(s_{Q_{A}}\right)
$$

$$
R_{2} \equiv\left(Q_{A}-Q_{B}\right)\left(P_{A}-P_{B}\right) \in \tau c\left(s_{P_{A}}\right) .
$$

We proceed by showing that the last two statements are equivalent. Indeed

$$
\begin{aligned}
R_{1} & =P_{A} Q_{A}+P_{B} Q_{B}-P_{A} Q_{B}-P_{B} Q_{A} \\
& =-\mathbf{1}-\mathbf{1}-P_{A} Q_{B}-\left(P_{A} Q_{B}\right)^{-1}
\end{aligned}
$$

and as $s_{Q_{A}}\left(-P_{A} Q_{B} \psi, \varphi\right)=s_{Q_{B}}(\psi, \varphi)$ it follows that $-P_{A} Q_{B}$ and hence $R_{1}$ is positive and hermitian with respect to $s_{Q_{A}}$. Similarly $R_{2}=-2$ $-Q_{A} P_{B}-\left(Q_{A} P_{B}\right)^{-1}$ is positive and hermitian with respect to $S_{P_{A}}$. So $R_{1}$ and $R_{2}$ are of trace class if the sum of its eigenvalues exists. Now, if $\psi$ is an eigenvector of say $Q_{A} P_{B}$ with eigenvalue $\lambda$ then

$$
R_{2} \psi=\left(-2-\lambda-\lambda^{-1}\right) \psi \text {. }
$$

On the other hand then $P_{B} \psi$ will be eigenvector of $P_{A} Q_{B}$ because

$$
P_{A} Q_{B} P_{B} \psi=-P_{A} \psi=P_{B} Q_{B} P_{A} \psi=\frac{1}{\lambda} P_{B} \psi
$$

and

$$
R_{1} P_{B} \psi=\left(-2-\lambda-\lambda^{-1}\right) P_{B} \psi .
$$

So the eigenvalues of $R_{1}$ and $R_{2}$ are the same and

$$
R_{1} \in \tau c\left(s_{Q_{A}}\right) \Leftrightarrow R_{2} \in \tau c\left(s_{P_{A}}\right)
$$

Next we remark that if $N$ is a positive hermitian operator then $\left(N+N^{-1}-2\right) \in \tau c \Leftrightarrow N-1 \in \sigma c$.

To see this, if $\left\{n_{i}\right\}$ are the eigenvalues of $N$, note that for both conditions it is necessary that $n_{i} \rightarrow 1$, and that

$$
n_{i}+n_{i}^{-1}-2=\frac{\left(n_{i}-1\right)^{2}}{n_{i}} \text {. }
$$


We finish this discussion by saying that if an operator $N$ is positive and hermitian with respect to $s_{Q_{A}}$ then

$$
N \in \tau c\left(s_{Q_{A}}\right) \Leftrightarrow N \in \tau c\left(s_{A}\right) .
$$

Consider the operator $C=\mathbf{1}+\sqrt{\mathbf{1 + A ^ { - 2 }}}$.

Clearly $1 \leqq C \leqq 2$ so that both $C$ and $C^{-1}$ are bounded. As $Q_{A}=C A$ and so $s_{Q_{A}}(\psi, \varphi)=s_{A}(\sqrt{C} \psi, \sqrt{C} \varphi)$ it follows that the metrics induced by $s_{Q_{A}}$ and $s_{A}$ are equivalent.

We summarise the results in the following lemma.

Lemma 10. Let $A, B \in S$, and define the operators on $H$ :

$$
\begin{aligned}
& Q_{A}=A+A \sqrt{\mathbf{1 + A ^ { - 2 }}} \\
& P_{A}=A-A \sqrt{\mathbf{1 + A ^ { - 2 }}}
\end{aligned}
$$

and the operator on $H \oplus H$

$$
E_{A}=\left(\begin{array}{cc}
A & A \sqrt{\mathbf{1 + A ^ { - 2 }}} \\
-A \sqrt{\mathbf{1 + A ^ { - 2 }}} & -A
\end{array}\right)
$$

and similarily for $B$.

Then the following statements are equivalent.

i) $\left(E_{A}-E_{B}\right)^{2} \in \tau c\left(s_{E_{A}}\right)$,

ii) $E_{A}-E_{B} \in \sigma c\left(s_{E_{A}}\right)$,

iii) $\left(P_{A}-P_{B}\right)\left(Q_{A}-Q_{B}\right) \in \tau c\left(s_{Q_{A}}\right)$,

iv) $\left(Q_{A}-Q_{B}\right)\left(P_{A}-P_{B}\right) \in \tau c\left(s_{P A}\right)$,

v) $\left(P_{A}-P_{B}\right)\left(Q_{A}-Q_{B}\right) \in \tau c\left(s_{A}\right)$

vi) $\left(Q_{A}^{-1} Q_{B}-1\right) \in \sigma c\left(s_{A}\right)$,

vii) $A^{-1}\left(Q_{B}-Q_{A}\right) \in \sigma c\left(s_{A}\right)$.

Proof. We already investigated the equivalences

$$
\text { (i) } \Leftrightarrow \quad \text { iii) } \Leftrightarrow \quad \text { iv) } \Leftrightarrow \quad \text { v) }
$$

We also showed that

$$
\begin{aligned}
\left(E_{A}-E_{B}\right)^{2}=-2- & E_{A} E_{B}-\left(E_{A} E_{B}\right)^{-1} \in \tau c\left(s_{E_{A}}\right) \\
& \Leftrightarrow-1-E_{A} E_{B}=E_{A}\left(E_{A}-E_{B}\right) \in \sigma c\left(s_{E_{A}}\right)
\end{aligned}
$$

and as $E_{A}$ is unitary with respect to $s_{E_{A}}$ this is equivalent with $E_{A}-E_{B} \in \tau c\left(s_{E_{A}}\right)$ proving (i) $\Leftrightarrow$ (ii).

To prove the equivalence of vi) with v) remark that

$$
\left(P_{A}-P_{B}\right)\left(Q_{A}-Q_{B}\right)=-2-P_{A} Q_{B}-\left(P_{A} Q_{B}\right)^{-1}
$$

and that

$$
1+P_{A} Q_{B}=1-Q_{A}^{-1} Q_{B} .
$$


To prove the equivalence of vi) and vii) remark that $Q_{A}=C A$ and so $A^{-1}\left(Q_{B}-Q_{A}\right)=C\left(Q_{A}^{-1} Q_{B}-1\right)$ and that both $C$ and $C^{-1}$ are bounded.

We next prove that if $A-B$ is of finite rank, then condition vii) of the previous lemma is fulfilled so that the following is true.

Lemma 11. If $A, B \in S$ and $A-B$ is of finite rank, then

$$
\left(E_{A}-E_{B}\right)^{2} \in \tau c\left(s_{E_{A}}\right) \text {. }
$$

Proof. If we denote by $X=A-B, Y=A \sqrt{\mathbf{1 + A ^ { - 2 }}}-B \sqrt{\mathbf{1 + B ^ { - 2 }}}$ then $Y=A\left(\sqrt{\mathbf{1 + A ^ { - 2 }}}-\sqrt{\mathbf{1 + B ^ { - 2 }}}\right)+X \sqrt{\mathbf{1 + B ^ { - 2 }}}$ so that

$$
A^{-1}\left(Q_{B}-Q_{A}\right)=\sqrt{1+A^{-2}}-\sqrt{1+B^{-2}}+A^{-1}\left(X+X \sqrt{1+B^{-2}}\right)
$$

and as $X$ is finite rank, condition vii) will be fulfilled if

$$
\left(\sqrt{\mathbf{1}+A^{-2}}-\sqrt{\mathbf{1 + B ^ { - 2 }}}\right) \in \sigma c\left(s_{A}\right) .
$$

Define $P=\mathbf{1}+A^{-2}$ and $Q=\mathbf{1}+B^{-2}$.

It remains to show that $\sqrt{P}-\sqrt{Q} \in \sigma c\left(s_{A}\right)$.

Remark that $A^{2}-B^{2}=A(A-B)+(A-B) B$ and that $A^{-2}-B^{-2}$ $=B^{-2}\left(A^{2}-B^{2}\right) A^{-2}$ so that $P-Q$ is finite rank. Furthermore $\sqrt{P}$ is positive and hermitian with respect to $s_{A}$ and so is $\sqrt{Q}$ with respect to $s_{B}$. By $s_{A}\left(A^{-1} B \sqrt{Q} \psi, \varphi\right)=s_{B}(\sqrt{Q} \psi, \varphi)$ it follows that $A^{-1} B \sqrt{Q}$ is positive and hermitian with respect to $S_{A}$.

By the same arguments as used in Lemma 4.1 of [6] it is true that $\sqrt{P}-\sqrt{Q}$ is compact and as $A^{-1} B \sqrt{Q}-\sqrt{Q}=-A^{-1} X \sqrt{Q}$ is finite rank $\sqrt{P}-A^{-1} B \sqrt{Q}$ is compact. If we define $S=\sqrt{\mathrm{P}}-A^{-1} B \sqrt{Q}$, $T=\sqrt{P}+A^{-1} B \sqrt{Q}, S^{\prime}=\sqrt{P}-\sqrt{Q}, T^{\prime}=\sqrt{P}+\sqrt{Q}$ then $S-S^{\prime}$ and $T-T^{\prime}$ are finite rank and $\frac{1}{2}\left(S^{\prime} T^{\prime}+T^{\prime} S^{\prime}\right)=P-Q$. So $\frac{1}{2}\left(S^{\prime} T^{\prime}+T^{\prime} S^{\prime}\right)$ and hence $\frac{1}{2}(S T+T S)$ is trace class and by the same arguments of Lemma 4.1 of [6], $S$ is Hilbert Schmidt and $S^{\prime}$ is Hilbert Schmidt. This completes the proof.

\section{Quasi-Equivalence of Quasi-Free States}

In this section we prove the main theorem on quasi-equivalence.

Consider the set $\left\{H_{\alpha}\right\}_{\alpha \in I}$ of all finite regular subspaces of $H$.

Then $\mathscr{J}=\left\{\mathscr{A}_{\alpha}=\overline{\Delta\left(H_{\alpha}, \sigma\right)}\right\}_{\alpha \in I}$ is a net of Weyl-subalgebra's of $\mathscr{A} \equiv \overline{\Delta(H, \sigma)}$ satisfying

i) to all pairs $\mathscr{A}_{\alpha}, \mathscr{A}_{\beta} \in \mathscr{J}$ there is a $\mathscr{A}_{\gamma} \in \mathscr{J}$ with $\mathscr{A}_{\alpha} \cup \mathscr{A}_{\beta} \subset \mathscr{A}_{\gamma}$,

ii) the unit of $\mathscr{A}=\overline{\mathbb{( H , \sigma )}}$ is contained in all $\mathscr{A}_{\alpha} \in \mathscr{J}$,

iii) the union $\bigcup_{\alpha \in I} \mathscr{A}_{\alpha}$ is norm dense in $\mathscr{A}$,

iv) every $\mathscr{A}_{\alpha} \in \mathscr{J}$ is type $I$. 
The last statement follows from the theorem of Von Neumann [1] saying that every representation of the Weyl algebra built on a finite space is a multiple of the Schrödinger representation which is irreducible. Hence every representation is type $I$. It then follows from a theorem of Haag, Kastler and Kadison [9, prop 13] that two quasi-free factor states $\omega_{A}$ and $\omega_{B}$ are quasi-equivalent iff to each $\varepsilon>0$ there is a $\mathscr{A}_{\alpha} \in \mathscr{J}$ such that $\left\|\left(\omega_{A}-\omega_{B}\right) \mid \mathscr{A}_{\alpha}^{c}\right\|<\varepsilon$ where $\mathscr{A}_{\alpha}^{c}$ is the commutant of $\mathscr{A}_{\alpha}$ in $\mathscr{A}$. Note that quasi-free states are locally normal.

We now show that $\mathscr{A}_{\alpha}^{c}=\overline{\Delta\left(H_{0}, \sigma\right)^{c}}=\overline{\Delta\left(H_{0}^{\perp}, \sigma\right)}$ where $H_{0}^{\perp}$ is the orthogonal completement of $H_{0}$ in $H$ with respect to $\sigma$.

Lemma 12. If $H_{0}$ is a regular subspace and finite, then

$$
\overline{\Delta\left(H_{0}, \sigma\right)^{c}}=\overline{\Delta\left(H_{0}^{\perp}, \sigma\right)}
$$

Proof. As $\overline{\Delta\left(H_{0}, \sigma\right)}$ and $\overline{\Delta\left(H_{0}^{\perp}, \sigma\right)}$ are generated by resp. $\Delta\left(H_{0}, \sigma\right)$ and $\Delta\left(H_{0}^{\perp}, \sigma\right)$ and as the last two algebra's commute, it follows that

$$
\overline{\Delta\left(H_{0}^{\perp}, \sigma\right)} \subseteq \overline{\Delta\left(H_{0}, \sigma\right)^{c}} .
$$

Therefore it is sufficient to show that for any $\varepsilon>0$ and $x \in \overline{\Delta\left(H_{0}, \sigma\right)^{c}}$ there exists a $y \in \Delta\left(H_{0}^{\perp}, \sigma\right)$ such that $\|y-x\|<\varepsilon$, since this means that $\Delta\left(H_{0}^{\perp}, \sigma\right)$ is dense in $\overline{\Delta\left(H_{0}, \sigma\right)^{c}}$ and hence $\overline{\Delta\left(H_{0}^{\perp}, \sigma\right)} \supseteqq \overline{\Delta\left(H_{0}, \sigma\right)^{c}}$.

So we consider $\varepsilon>0$ and $x \in \overline{\Delta\left(H_{0}, \sigma\right)^{c}}$ and we construct such a $y$. As $\Delta(H, \sigma)$ is dense in $\overline{\Delta(H, \sigma)}$ there exists an element of the form

$$
z=\sum_{i=1}^{p} a_{i} \delta_{\psi_{i}}, \psi_{i} \in H, \quad \text { such that } \quad\|z-x\|<\varepsilon / 2
$$

$z$ can be written as $z=z^{\perp}+z_{1}$ where

$$
\begin{aligned}
& z^{\perp}=\Sigma a_{i} \delta_{\psi_{i}} \quad \text { with } \quad \psi_{i} \in H_{0}^{\perp} \\
& z_{1}=\Sigma a_{i} \delta_{\psi_{i}^{\perp}} \delta_{\varphi_{i}} \quad \text { with } \quad \psi_{i}^{\perp} \in H_{0}^{\perp} \quad \text { and } \quad \varphi_{i} \neq \mathbf{0} \in H_{0} .
\end{aligned}
$$

Next for any integer $n$ we define the map

$$
u \in \overline{\Delta(H, \sigma)} \rightarrow \tau_{n}(u)=\frac{1}{\pi^{n_{0}}} \int_{H_{0}} \exp \left(-S_{J}(\psi, \psi)\right) \delta_{n \psi} u \delta_{-n \psi} d \psi
$$

where $2 n_{0}$ is the dimension of $H_{0}, J$ a complex structure leaving $H_{0}$ invariant and $d \psi$ the Lebesque measure on $H_{0}$ induced by the scalar product $s_{J}$.

Clearly if $u \in{\overline{\Delta\left(H_{0}, \sigma\right)^{c}}}^{c}$ then

$$
\tau_{n}(u)=u \frac{1}{\pi^{n_{0}}} \int_{\psi \in H_{0}} \exp \left(-S_{J}(\psi, \psi)\right) d \psi=u
$$

so that $\tau_{n}(x)=x$. 
Next compute $\tau_{n}\left(\delta_{\varphi_{t}}\right)$ with $\varphi_{i} \in H_{0}$.

By a straightforward application of the multiplication rules

$$
\begin{aligned}
\tau_{n}\left(\delta_{\varphi_{i}}\right) & =\frac{1}{\pi^{n_{0}}} \int_{\psi \in H_{0}} e^{-s_{J}(\psi, \psi)} e^{-2 i n \sigma\left(\psi, \varphi_{\imath}\right)} \delta_{\varphi_{i}} d \psi \\
& =\delta_{\varphi_{i}} \frac{1}{\pi^{n_{0}}} \int_{\psi^{\prime} \in H_{0}} e^{-s_{J}\left(\psi^{\prime}, \psi^{\prime}\right)} e^{-2 i n s_{J}\left(\psi^{\prime}, \varphi_{i}\right)} d \psi^{\prime}
\end{aligned}
$$

as $J$ is an isometry (with $\psi^{\prime}=J \psi$ ).

By a straightforward extension of the formula

$$
\frac{1}{\sqrt{\pi}} \int_{-\infty}^{+\infty} \exp \left(-x^{2}-2 n i y x\right) d x=\exp \left(-n^{2} y^{2}\right)
$$

we get

$$
\tau_{n}\left(\delta_{\varphi_{i}}\right)=\exp \left(-n^{2} S_{J}\left(\varphi_{i}, \varphi_{i}\right)\right) \delta_{\varphi_{i}} .
$$

If we apply this to $\tau_{n}\left(z_{1}\right)$ we find

$$
\tau_{n}\left(z_{1}\right)=\Sigma a_{i} \delta_{\psi_{i}^{\perp}} \delta_{\varphi_{i}} \exp \left(-n^{2} s_{J}\left(\varphi_{i}, \varphi_{i}\right)\right)
$$

and the norm $\left\|\tau_{n}\left(z_{1}\right)\right\|$ is smaller than

$$
\Sigma\left|a_{i}\right| \exp \left(-n^{2} s_{J}\left(\varphi_{i}, \varphi_{i}\right)\right)\left\|\delta_{\psi_{i}^{\perp}}\right\| \cdot\left\|\delta_{\varphi_{i}}\right\|=\Sigma\left|a_{i}\right| \exp \left(-n^{2} s_{J}\left(\varphi_{i}, \varphi_{i}\right)\right)
$$

which, for a fixed element $z$ can be made smaller than $\varepsilon / 2$ for large $n$, as $\varphi_{i} \neq 0$.

We summarize the result by considering

$$
\left\|x-z^{\perp}\right\|=\left\|\tau_{n}(x)-\tau_{n}(z)+\tau_{n}\left(z_{1}\right)\right\| .
$$

So

$$
\begin{aligned}
\left\|x-z^{\perp}\right\| & \leqq\left\|\tau_{n}(x-z)\right\|+\left\|\tau_{n}\left(z_{1}\right)\right\| \\
& \leqq \frac{1}{\pi^{n_{0}}} \int e^{-s_{J}(\psi, \psi)}\left\|\delta_{n \psi}\right\|\|x-z\|\left\|\delta_{-n \psi}\right\| d \psi+\varepsilon / 2 \\
& \leqq \frac{1}{\pi^{n_{0}}} \int e^{-s_{J}(\psi, \psi)} d \psi \cdot \varepsilon / 2+\varepsilon / 2, \\
& =\varepsilon
\end{aligned}
$$

This completes the proof.

As a result of Lemma 12 we find that two quasi-free factor states $\omega_{A}$ and $\omega_{B}$ are quasi-equivalent if and only if there is a finite regular subspace $H_{\alpha}$ of $H$ for every $\varepsilon>0$ such that

$$
\left\|\left(\omega_{A}-\omega_{B}\right) \mid \overline{\Delta\left(H_{\alpha}^{\perp}, \sigma\right)}\right\|<\varepsilon .
$$


We use this in the following theorem. Note that the quasi-free state $\omega_{A}, A \in S$ is a factor state if and only if the norm continuous extension of $\sigma$ to the completion $\bar{H}^{s_{A}}$ of $H$ with respect to $s_{A}$ is non-degenerate [5].

Theorem. Two quasi-free factor states $\omega_{A}$ and $\omega_{B}$ with $A, B \in S$ are quasi-equivalent if and only if $\left(Q_{A}^{-1} Q_{B}-1\right) \in \sigma c\left(s_{A}\right)$.

Proof. The proof is completely the same as the one of Theorem 5.1 of $[6]$.

Suppose first that $\left(Q_{A}^{-1} Q_{B}-1\right) \in \sigma c\left(s_{A}\right)$, then by Lemma 10 $\left(E_{A}-E_{B}\right)^{2} \in \tau c\left(s_{E_{A}}\right)$ and therefore $\omega_{E_{A}}$ and $\omega_{E B}$ are unitarily equivalent [7]. By the work of Haag, Kadison and Kastler, for any $\varepsilon>0$ there is a finite regular subspace $K$ of $H \oplus H$ such that

$$
\left\|\left(\omega_{E_{A}}-\omega_{E_{B}}\right) \mid \overline{\Delta\left(K^{\perp}, \sigma \oplus-\sigma\right)}\right\|<\varepsilon .
$$

To every finite subspace $K$ of $H \oplus H$ there exists a finite regular subspace $H_{0}$ of $H$ such that $K \subset H_{0} \oplus H_{0}$ and therefore $H_{0}^{\perp} \oplus H_{0}^{\perp} \subset K^{\perp}$. With the same arguments as used in section 4 then

$$
\begin{aligned}
\left\|\left(\omega_{A}-\omega_{B}\right) \mid \overline{\Delta\left(H_{0}^{\perp}, \sigma\right)}\right\| & \leqq\left\|\left(\omega_{E_{A}}-\omega_{E_{B}}\right) \mid \overline{\Delta\left(H_{0}^{\perp} \oplus H_{0}^{\perp}, \sigma \oplus-\sigma\right)}\right\| \\
& \leqq\left\|\left(\omega_{E_{A}}-\omega_{E_{B}}\right) \mid \overline{\Delta\left(K^{\perp}, \sigma \oplus-\sigma\right)}\right\| \\
& \leqq \varepsilon
\end{aligned}
$$

So $\omega_{A}$ and $\omega_{B}$ are quasi-equivalent.

Conversely, suppose that $\omega_{A}$ and $\omega_{B}$ are quasi-equivalent. Then there is a finite regular subspace $H_{0}$ of $H$ for any $\varepsilon>0$ such that

$$
\left\|\left(\omega_{A}-\omega_{B}\right) \mid \overline{\Delta\left(H_{0}^{\perp}, \sigma\right)}\right\|<\varepsilon,
$$

As in [6], let $E$ be the orthogonal projection on $H_{0}$.

Define $A_{1}=E A E+(\mathbf{1}-E) A(\mathbf{1}-E)$ and $B_{1}=E A E+(\mathbf{1}-E) B(\mathbf{1}-E)$.

In the proof of Lemma 8 we showed that if, $A, B \in S$ and $E$ is the orthogonal projection on a subspace $H_{0}$, then $E A E \in S$. Similarly $(1-E) B(1-E) \in S$ and $(1-E) A(1-E) \in S$. Moreover it is possible to conclude that also $A_{1} \in S$ and $B_{1} \in S$.

Clearly $A-A_{1}$ and $B-B_{1}$ have finite rank and hence by Lemma 11 $E_{A}-E_{A_{1}}$ and $E_{B}-E_{B_{1}}$ are Hilbert Schmidt and so $\omega_{E_{A}} \sim \omega_{E_{A_{1}}}$ and $\omega_{E_{B}} \sim \omega_{E_{B_{1}}}$ [7]. On the other hand, as $A_{1}$ coincides with $A$ on $H_{0}^{\perp}$ and similarly for $B$ we find that

$$
\left\|\left(\omega_{A_{1}}-\omega_{B_{1}}\right) \mid \overline{\Delta\left(H_{0}^{\perp}, \sigma\right)}\right\| \subset \varepsilon .
$$

Moreover $\omega_{A_{1}}$ and $\omega_{B_{1}}$ are product states and coincide on $\overline{\Delta\left(H_{0}, \sigma\right)}$ so

$$
\text { that }\left\|\omega_{A_{1}}-\omega_{B_{1}}\right\|<\varepsilon \text {. }
$$


Then by Lemma 9

$$
\left\|\omega_{E_{A_{1}}}-\omega_{E_{B_{1}}}\right\| \leqq 2\left\|\omega_{A_{1}}-\omega_{B_{1}}\right\|^{\frac{1}{2}} \leqq 2 \varepsilon^{\frac{1}{2}}
$$

and so $\omega_{E_{A_{1}}} \sim \omega_{E_{B_{1}}}$ [9]. Consequently $\omega_{E_{A}} \sim \omega_{E_{B}}$ and by [7]:

$$
\left(E_{A}-E_{B}\right)^{2} \in \tau c\left(s_{E_{A}}\right) .
$$

Finally the use of Lemma 10 completes the proof.

Remark. Lemma 10 provides us a large number of equivalent conditions. If the states $\omega_{A}$ and $\omega_{B}$ are pure states and so $A^{2}=B^{2}=-\mathbf{1}$ then $Q_{A}=P_{A}=A$ and the conditions i), iii), iv) and v) are essentially $(A-B)^{2} \in \tau c\left(s_{A}\right)$. On the other hand conditions ii), vi) and vii) state $A-B \in \sigma c\left(s_{A}\right)$.

\title{
References
}

1. Neumann, J.von: Math. Ann. 104, 570 (1931).

2. Kastler, D.: Commun. math. Phys. 1, 14 (1965).

3. Manuceau, J.: Ann. Inst. Henri Poincaré 8, 139 (1968).

4. Robinson, D. W.: Commun. math. Phys. 1, 159 (1965).

5. Manuceau, J., Verbeure, A.: Commun. math. Phys. 9, 293 (1968).

6. Powers, R. T., Størmer, E.: Commun. math. Phys. 16, 1 (1970).

7. Van Daele,A., Verbeure,A.: Unitary equivalence of Fock representations on the Weyl algebra: Commun. math. Phys. 20, 268-278 (1971).

8. Verbeure, A.: Private communication.

9. Haag, R., Kadison, R. V., Kastler, D.: Commun. math. Phys. 16, 81 (1970).

10. Muray, F. J., Neumann, J.von: Ann. Math. 37, 116 (1936).

\author{
A. Van Daele \\ Universitetet I Oslo \\ Matematisk Institutt, Postboks 1053 \\ Blindern - Oslo 3 \\ Norway
}

